\section{Sex-dependent membranopathy in stored human red blood cells}

Over the past years, concern has been raised over the impact of red blood cell (RBC) alterations, related to prolonged hypothermic storage, on patient safety. Multiple reviews and works focus on presentation of a full list of changes characteristic for $\mathrm{RBC}$ storage lesions including alterations in RBC metabolomics, cytosol structure and membrane organization. ${ }^{1-6}$ We recently showed that a decrease in RBC deformability was observed as a relative- ly late consequence of packed $\mathrm{RBC}$ ( $\mathrm{pRBC}$ ) storage, while the analysis of vesiculation observed on the RBC membranes in nanoscale with the application of atomic force microscopy (AFM) supported by analysis of biochemical parameters allowed for the detection of early changes. ${ }^{7}$ However, these results, as well as those of other groups were mainly obtained from $\mathrm{pRBC}$ of male donors, or the sex of donors was not specified. ${ }^{5,8}$ Recently, it has also been suggested that RBC from females are less prone to storage lesion and age slower than male erythrocytes ${ }^{9,10}$ but these reports were mainly focused on metabolic and functional RBC analysis.
A
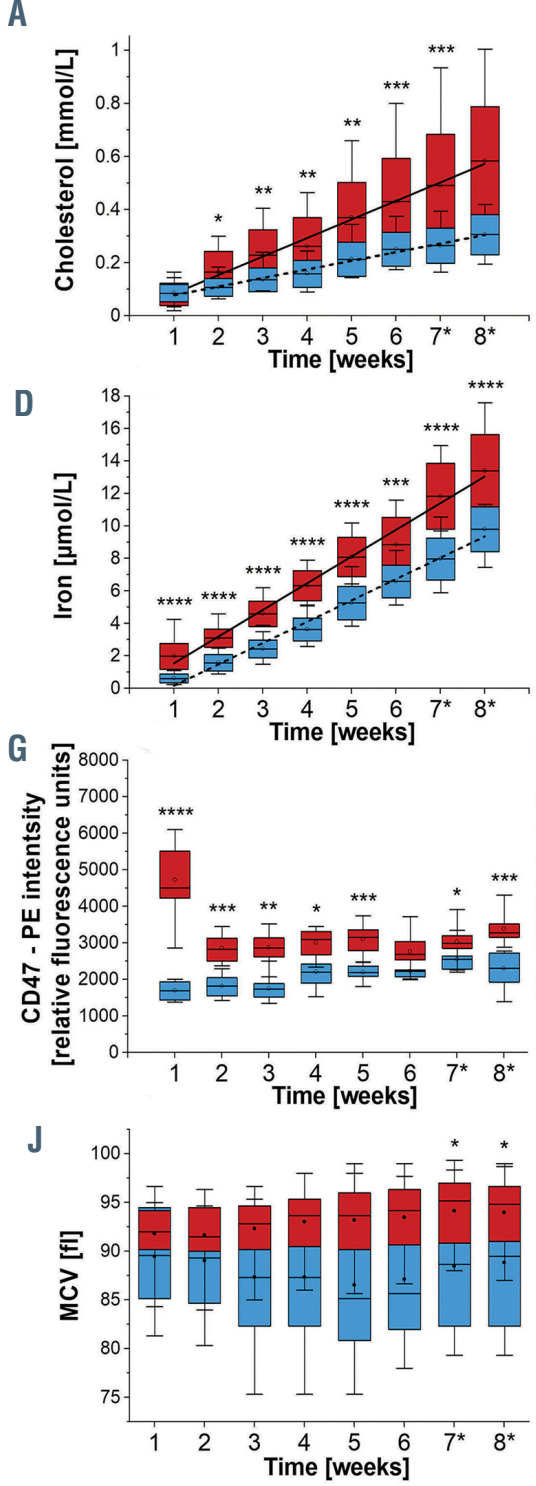

B
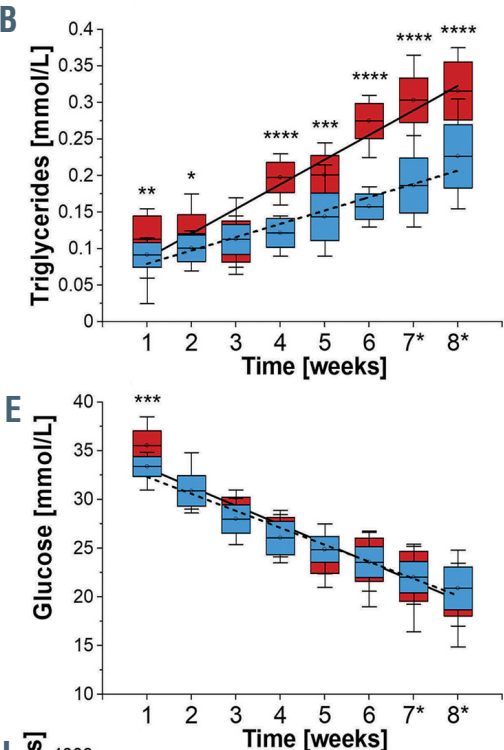

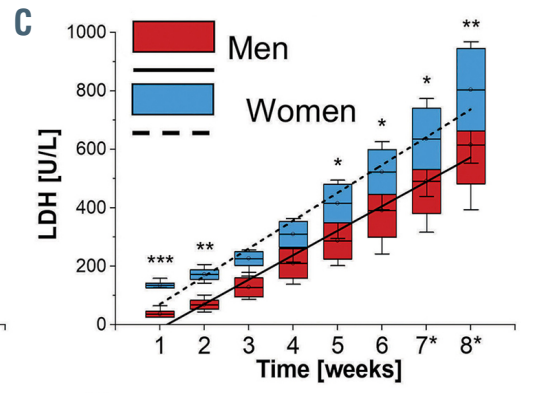

F
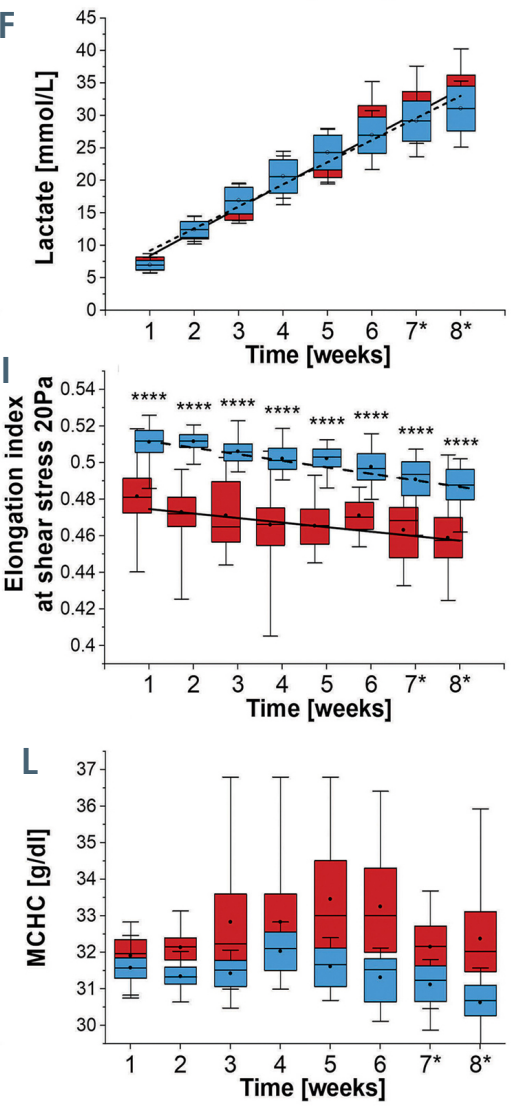

Figure 1. Storage time-dependent changes in biochemical, morphological and functional red blood cell parameters. Biochemical parameters: (A) cholesterol, (B) triglycerides, (C) lactate dehydrogenase (LDH), (D) free iron of human packed red blood cells (pRBC), (E) glucose, (F) lactate; chosen protein expression: (G) CD47 and (H) phosphatidylserine (PS); (I) pRBC deformability; the blood count including: (J) mean corpuscular volume (MCV), (K) hematocrit (HCT), (L) mean corpuscular hemoglobin concentration (MCHC). The blood was withdrawn from men $(n=12)$ and women ( $n=12)$. Results (A to $F)$ were obtained from pRBC supernatant with ABX Pentra 400 (Horiba Medical, Japan) are given as mean \pm standard deviation (SD), data distribution is presented as box plots (mean value, mean $\pm \mathrm{SD}$, min-max whiskers). Results (G to L) presented as box plots (median, Q1, Q3, interquartile range, min-max whiskers). Q1, Q3 indicate $25^{\text {th }}$ and $75^{\text {th }}$ percentiles, respectively. Results ( $\mathrm{G}$ and $\mathrm{H}$ ) were obtained using a mixture of mouse anti-human antibodies carrying fluorescent markers: CD45 - APC-Cy7 (1:200, BD cat. no. 348815), CD71 - APC (1:200, BD cat. no. 551374), CD47 - PE (1:200, BD cat. no. 556046) and annexin V - FITC (1:200, BD cat. no. 556547). Cellular analysis was performed with LSRII flow cytometer (BD Biosciences). RBC deformability was studied at $37^{\circ} \mathrm{C}$ as elongation index at shear rates of $20 \mathrm{~Pa}$ with the use of ektacytometer RheoScanAnD 300 (RheoMeditech, Seoul, Korea) while parameters (J and L) with hematology analyzer Abc Vet (Horiba Medical, France). Data (A to L) normality was assessed using Shapiro-Wilk test. The significance of the differences between men and women in each week was evaluated by One-Way ANOVA with Tukey's test. If data distribution was not normal, non-parametric Kruskal-Wallis test with the Dunn's post hoc test if appropriate; $* P<0.05, * * P<0.01, * * * P<0.001 * * * * P<0.0001$ indicates significant difference between men and women in each week. 
A

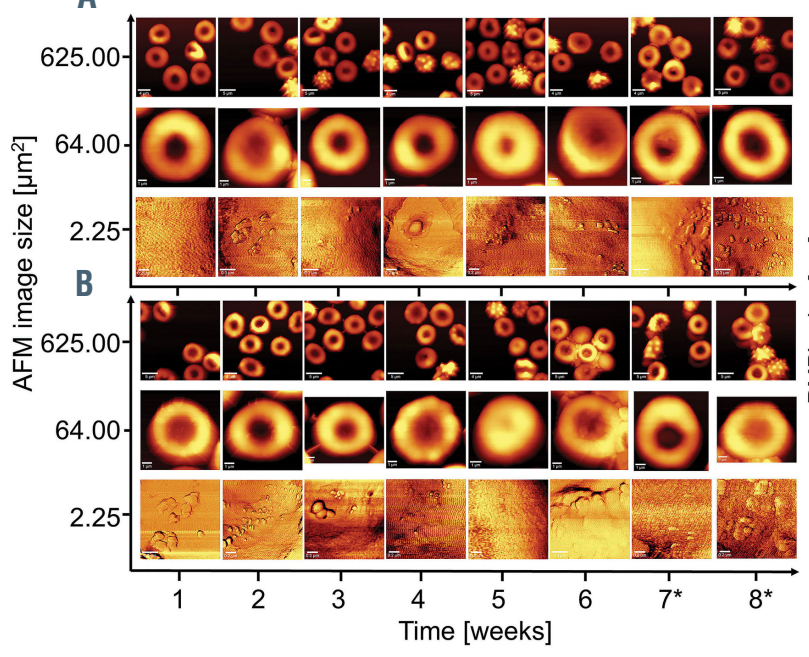

B

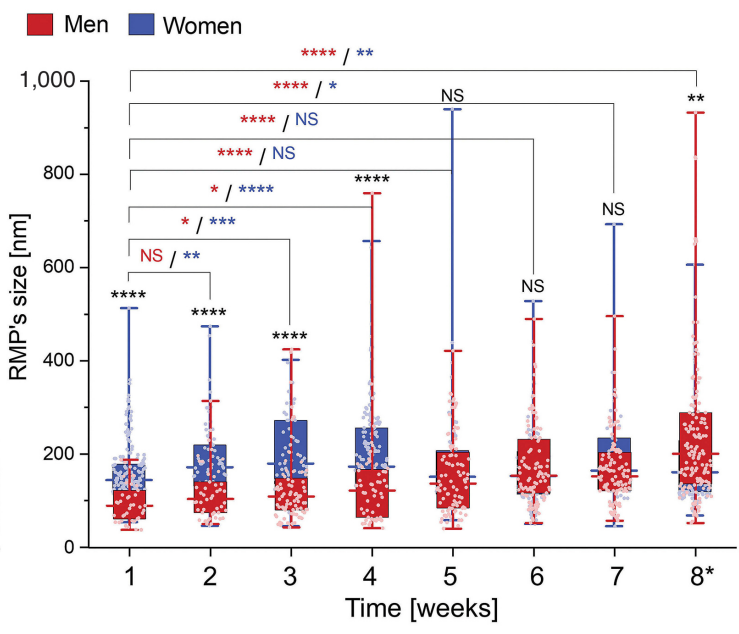

Figure 2. Time sequence of the appearance of red blood cell (RBC)-derived microparticles on the surface of human packed RBC isolated from (A) men and (B) women. Examples of atomic force microscopy (AFM) images with the use of WITec confocal CRM alpha 300 in non-contact mode (AC) (WITec, UIm, Germany) and dry Zeiss objective (ECEPIPLAN 20x/0.4). AFM images of 256x256 lines and 512x512 lines were collected from areas of $25 \times 25 u m^{2}, 8 \times 8 u m^{2}$ and $1.5 \times 1.5$ $\mu \mathrm{m}^{2}$, which were performed at room temperature on dried smears of (A) female RBC and (B) male RBC fixed with $1 \%$ glutaraldehyde (10 minutes). (C) Sex-specific, time-dependent changes of the RBC-derived microparticle (RMP) sizes observed on the surface of human packed RBC. Data distribution is presented as box plots (median, Q1, Q3, interquartile range, min-max whiskers). Q1, Q3 indicate $25^{\text {th }}$ and $75^{\text {th }}$ percentiles, respectively. ${ }^{*}$ Weeks 7 and 8 are additional measurements exceeding expiration date. Statistical significance of the obtained results $(n>35)$ was tested with Kruskal-Wallis ANOVA non-parametric test followed by Tukey's post hoc. NS: not significant; $* P<0.05 ; * * P<0.01, * * * P<0.001, * * * * P<0.0001$ ).

In the present study, we investigated the influence of donor's sex on the sequence of changes observed during long-term storage of leukocyto-depleted pRBC containing SAGM (saline, adenine, glucose, mannitol) additive solution and a trace amount of CPD (citrate, phosphate, dextrose) preservative, which were purchased from the Regional Center for Blood Donation and Hemotherapy in Krakow, Poland. According to the principles outlined in the World Medical Association Declaration of Helsinki, as well as a Bioethical Commission of the Jagiellonian University, venous blood for $\mathrm{pRBC}$ was obtained from volunteers including men $(\mathrm{mRBC}) \mathrm{n}=12$, aged $<30$ years $(n=3), 30-39$ years $(n=4),>40$ years $(n=5)$ and women $(\mathrm{fRBC}) \mathrm{n}=12$, aged $<30$ years $(\mathrm{n}=4), 30-39$ years $(\mathrm{n}=4)$, $>40$ years $(n=4)$. All analyzes were carried out weekly throughout 6 weeks of pRBC storage, while the seventh and eighth week's measurements were designed as additional time points exceeding the pRBC expiration date (42 days), and were focused on the membranopathy on the level of $\mathrm{pRBC}$ membrane biochemistry, physical and mechanical properties (Figure 1), as well nanoscale changes (Figure 2).

Throughout the storage time, statistically significant differences between $\mathrm{mRBC}$ and $\mathrm{ARBC}$ in the kinetics of cholesterol and triglycerides' increase were observed (Figure 1A and B). An increase between the first and eighth week was 2.27 times greater for cholesterol and 1.43 times greater for triglycerides in case of male donors. Such an increase in the lipid fraction in the sheathing solution during $\mathrm{pRBC}$ storage is related to lipidome alterations and disruption of phospholipid asymmetry in the RBC membrane. ${ }^{11}$ Our recent work and results of other groups suggest that the release of lipids from RBC membranes can be correlated with red blood cell-derived microparticle (RMP) formation. ${ }^{12}$ Our results agree with previous reports showing that the cholesterol level in fresh sample is similar in both sexes, while triglyceride level is lower in women. ${ }^{13,14}$

The levels of free iron, a renown and specific indicator of hemolysis, were higher in $\mathrm{mRBC}$, suggesting greater hemolysis rate (Figure 1D), what agrees with previous results. ${ }^{4,10}$ Lactate dehydrogenase (LDH) levels in both males and females below 40 years old did not differ, but higher average levels (Figure 1C) of LDH in fRBC could originate from higher skeletal muscle damage in postmenopausal women ${ }^{15}$ (age $>40$ years old; Online Supplementary Figure S1E). Changes in glucose and lactic acid concentration (Figure $1 \mathrm{E}$ and $\mathrm{F}$ ), which are a natural consequence of the glycolysis pathway, didn't show any sex-driven divergence. Additionally, some variations in individual values of glucose, lactate and free iron were observed in the youngest donors $(<30$ years old), while cholesterol levels were most variable in patients aged 30-39 years (Online Supplementary Figure S1C). The variation in the youngest blood donors may be related to a previously reported "healthy donor effect". ${ }^{16}$

Anti-CD 45 labeling proved the purity of pRBC, showing only $0.1-0.2 \%$ of the whole cell population to be CD 45-positive. From around fourth to fifth week of storage the expression of CD45 and CD71 (marker of reticulocytes) started to increase in $\mathrm{MRBC}$ compared to fRBC (Online Supplementary Figure S2). This could be a result of faster maturation of reticulocyte and/or leukocyte leftovers maturation in $\mathrm{mRBC}$ during storage. In case of both CD45 and CD71, minor changes were observed regardless of the donors' age. The level of CD47 ('don't eat me' signal) expression on RBC membranes differed significantly between $\mathrm{mRBC}$ and $\mathrm{FRBC}$ throughout the storage period (Figure 1G). Over time, it decreased age-independently in $\mathrm{mRBC}$, but did not change significantly in fRBC (Online Supplementary Figure S2). Exposure of CD47 was previously found as a result of storage-dependent proteolytic cleavage, oxidation and/or conformational changes caused by rearrangements of the phospholipid bilayer. ${ }^{11}$ Our results of CD47 expression analysis confirm conformational changes of RBC membrane and phospholipid bilayer destabilization in $\mathrm{mRBC}$ that lead to erythrocyte 


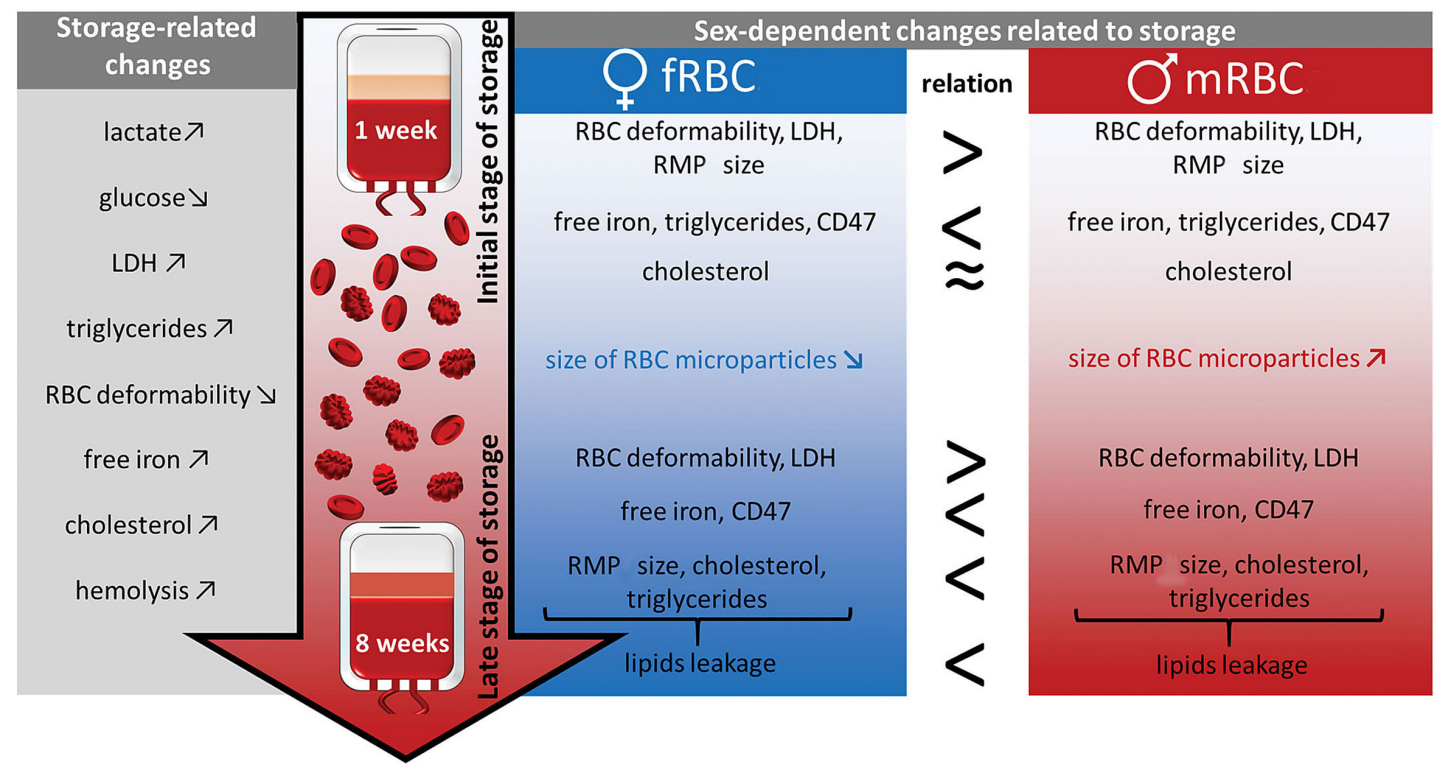

Figure 3. Schematic summary of the sex-specific, time-dependent changes in packed red blood cells derived from men ( $\mathrm{n}=12$ ) and women ( $\mathrm{n}=12$ ). Packed red blood cells (pRBC) were cold-stored for 8 weeks (weeks 7 and 8 being additional time points exceeding expiration date). Physiological parameters were assessed with use of biochemical analysis, morphological analysis, flow cytometry, ektacytometry and atomic force microscopy. fRBC: female RBC; mRBC: male RBC; LDH: lactate dehydrogenase; RMP: red blood cell-derived mircoparticle.

clearance $^{17}$ and proove that basal composition of RBC membrane is sex specific.

Expression of phosphatidylserine (PS, a marker of senescence or damage) was found to increase during $\mathrm{fRBC}$ and $\mathrm{mRBC}$ storage ${ }^{18}$ but our results suggest that $\mathrm{mRBC}$ are more prone to undergo membranopathies that result in higher PS exposure, at least at some point of their lifespan (Figure 1H; Online Supplementary Figure S2). In both sexes the values of maximum deformability were decreasing age-independently at the same speed during storage, whereas $\mathrm{fRBC}$ retained higher deformability compared to $\mathrm{mRBC}$ at every time point of storage (Figure 1I; Online Supplementary Figure S3). These data agree with previous research, ${ }^{19}$ which showed lower RBC deformability in males. RBC, hemoglobin (HGB), hematocrit (HCT) and mean corpuscular hemoglobin concentration (MCHC) were significantly higher in $\mathrm{mRBC}$ compared to fRBC throughout entire storage time (Figure 1). No differences in mean corpuscular hemoglobin $(\mathrm{MCH})$ values between $\mathrm{mRBC}$ and $\mathrm{ARBCs}$ were detected. Mean corpuscular volume (MCV) (Figure 1J) was relatively stable up to the fifth week of storage, at which point we saw a significant increase in MCV only in $\mathrm{MRBC}$. This is indicative of greater swelling of $\mathrm{mRBC}$ during long-term storage, which is due to higher alterations in their membranes, as reflected by higher leakage of membrane lipid and their lower deformability. Apart from the MCV increase, a slight decrease in MCHC (Figure 1L) levels during storage of mRBC confirms cell membrane loss. ${ }^{20}$

In order to study the size of RMP appearing on the RBC surface in $\mathrm{mRBC}$ and $\mathrm{ARBC}$ during storage, we applied our recently published AFM methodology ${ }^{7}$ (Figure 2). Different changes in the RMP size were observed in two clear-cut time intervals. During the first interval lasting from the first to fourth week, fluctuation of the RMP mean size, oscillating around $150 \mathrm{~nm}$, was observed in $\mathrm{mRBC}$. In case of $\mathrm{ARBC}$ a different tendency was observed: the values representing RMP sizes significantly increased from around $150 \mathrm{~nm}$ in the first week, up to around $200 \mathrm{~nm}$ in the fourth week. During the second time interval, observed phenomena reversed in both sexes. In $\mathrm{mRBC}$, a significant increase in the values from around $150 \mathrm{~nm}$ up to $200 \mathrm{~nm}$ was observed, while in fRBC the RMP sizes stabilized on the level of approximately $160 \mathrm{~nm}$ until the end of the storage.

Our findings shed a new light on pRBC sex-derived differences in the performance during storage related to $\mathrm{RBC}$ membranopathy, which are summed up in Figure 3. To the best of our knowledge, for the first time we have presented significant sex-related differences between the kinetics of both cholesterol and triglyceride level changes observed in $\mathrm{mRBC}$ and $\mathrm{ARBC}$ in this work. Our results show that increased kinetics of RBC membrane lipid leakage is related with shedding of RMP of smaller size (approximately $150 \mathrm{~nm}$ ) until the fourth week of storage and bigger RMP (approximately $200 \mathrm{~nm}$ ) starting from the fifth week. On the other hand, our results reveal that shedding of large RMP until the fourth week of storage, seems to be an adaptation of $\mathrm{ARBC}$ to adverse conditions and demonstrates their higher resistance in comparison to $\mathrm{mRBC}$. This is reflected by a slower membrane lipid leakage and higher deformability of $\mathrm{FRBC}$ during the whole time of storage. A relation observed between membrane lipids leakage and RMP formation suggests that the mechanism of RMP shedding, RMP composition and $\mathrm{RBC}$ response to storage conditions are all sex-related. These changes are accompanied by a higher level of hemolysis and a decreased deformability at each time point of $\mathrm{pRBC}$ storage observed for male donors. A statistically significant increase in MCV and CD47 expression of $\mathrm{mRBC}$ in comparison to $\mathrm{fRBC}$ during prolonged storage confirmed stronger swelling of $\mathrm{mRBC}$, which originates from greater alterations of their membranes including rearrangements of the phospholipid bilayer. fRBC were shown to be more resilient to an unfavorable mileu which was reflected by their higher deformability, no changes in MCV, slower membrane lipid leakage and lower hemolysis. Cold storage seems to affect $\mathrm{mRBC}$ in a different, yet more severe way, while fRBC cope with adverse conditions more efficienty, a fact that should be 
considered by modern transfusion medicine. Results may have an impact on future studies regarding: the need for greater control over the process of selection, assignment, and administration of blood labile products. Moreover, they might be a call for re-evaluation and for the setting of new standards for the storage of blood products.

Ewa Szczesny-Malysiak, ${ }^{1}$ Tasnim Mohaissen, ${ }^{1,2}$

\section{Katarzyna Bulat, ${ }^{1}$ Magdalena Kaczmarska, ${ }^{1}$} Aleksandra Wajda ${ }^{1,3}$ and Katarzyna M. Marzec'

$1 J a g i e l l o n i a n$ Center for Experimental Therapeutics, Jagiellonian University; ${ }^{2}$ Faculty of Pharmacy, Jagiellonian University Medical College and ${ }^{3}$ Faculty of Materials Science and Ceramics, AGH University of Science and Technology, Krakow, Poland

Correspondence:

KATARZYNA MARIA MARZEC - katarzyna.marzec@jcet.eu doi:10.3324/haematol.2021.278895

Received: April 12, 2021.

Accepted: June 21, 2021.

Pre-published: July 8, 2021.

Disclosures: no conflicts of interest to disclose.

Contributions: ES- $M$ designed the study, collected flow cytometry data, analyzed and interpreted data, wrote and drafted the manuscript; $T M$ collected the ektacytometry data, analyzed and interpreted data and wrote the manuscript; KB collected biochemical data, analyzed and interpreted data; MK collected, analyzed and interpreted AFM data; AW prepared samples for all measurements, analyzed biochemical and morphological data; KMM developed the concept and design, provided funding, wrote and approved the final version to be published.

Acknowledgements: we would like to thank Mrs. Beata Mazurek from the Regional Center for Blood Donation and Haemotherapy in Krakow, who coordinated the donation and the preparation of $P R B C$ used in this study.

Funding: this work was supported by the National Center for Research and Development, Poland (LIDER/13/0076/L8/16/NCBR/2017). The open-access publication of this article was funded by the Priority Research Area BioS under the program "Excellence Initiative - Research University" at the Jagiellonian University in Krakow.

Data sharing statement: for original data please visit http://dx.doi.org/10.17632/dwxw2wwjtm.1

Research limitation: the experimental setup was based on twelve healthy male ( $<30$ years, $n=3 ; 30-39$ years, $n=4 ;>40$ years, $n=5)$ and twelve female donors $(<30$ years, $n=4 ; 30-39$ years, $n=4 ;>40$ years, $n=4)$ so age was evenly distributed in the range of $18-60$ years for $f R B C$ and $m R B C$. However, conclusions regarding comparison of specific age groups presented in the Online Supplementary Appendix should be drawn in relation to the relatively small number $(n=3-5)$. Observed processes may be an outcome of sex-related hormones action, which were not studied in this work. Major limitations of this study could be addressed in future research.

\section{References}

1. Yoshida T, Prudent M, D'Alessandro A. Red blood cell storage lesion: causes and potential clinical consequences. Blood Transfus. 2019;17(1):27-52

2. Szczesny-Malysiak E, Dybas J, Blat A, et al. Irreversible alterations in the hemoglobin structure affect oxygen binding in human packed red blood cells. Biochim Biophys Acta Mol Cell Res. 2020; 1867(11):118803.

3. Roubinian NH, Plimier C, Woo JP, et al. Effect of donor, component, and recipient characteristics on hemoglobin increments following red blood cell transfusion. Blood. 2019;134(13):1003-1013.

4. Kanias T, Lanteri MC, Page GP, et al. Ethnicity, sex, and age are determinants of red blood cell storage and stress hemolysis: results of the REDS-III RBC-Omics study. Blood Adv. 2017;1(15):11321141

5. Tzounakas VL, Georgatzakou HT, Kriebardis AG, et al. Donor variation effect on red blood cell storage lesion: a multivariable, yet consistent, story. Transfusion 2016;56(6):1274-1286.

6.Zimna A, Kaczmarska M, Szczesny-Malysiak E, et al. An insight into the stages of ion leakage during red blood cell storage. Int J Mol Sci. 2021;22(6):2885.

7. Kaczmarska M, Grosicki M, Bulat K, et al. Temporal sequence of the human RBCs' vesiculation observed in nano-scale with application of AFM and complementary techniques. Nanomedicine. 2020;28:102221.

8. D'Alessandro A, Gray AD, Szczepiorkowski ZM, Hansen K, Herschel LH, Dumont LJ. Red blood cell metabolic responses to refrigerated storage, rejuvenation, and frozen storage. Transfusion. 2017;57(4):1019-1030

9. D'Alessandro A, Fu X, Kanias T, et al. Donor sex, age and ethnicity impact stored red blood cell antioxidant metabolism through mechanisms in part explained by glucose 6-phosphate dehydrogenase levels and activity. Haematologica. 2021;106(5):1290-1302.

10. Mykhailova O, Olafson C, Turner TR, D’Alessandro A, Acker JP. Donor-dependent aging of young and old red blood cell subpopulations: Metabolic and functional heterogeneity. Transfusion. 2020;60(11):2633-2646.

11. Karon BS, Van Buskirk CM, Jaben EA, Hoyer JD, Thomas DD. Temporal sequence of major biochemical events during Blood Bank storage of packed red blood cells. Blood Transfus. 2012;10(4):453461.

12. Said AS, Rogers SC, Doctor A. Physiologic impact of circulating RBC microparticles upon blood-vascular interactions. Front Physiol. 2018;8:1120.

13. Theobald HE, Chowienczyk PJ, Whittall R, Humphries SE, Sanders TAB. LDL cholesterol-raising effect of low-dose docosahexaenoic acid in middle-aged men and women. Am J Clin Nutr. 2004;79(4):558-563.

14. Magkos F, Mittendorfer B. Gender differences in lipid metabolism and the effect of obesity. Obst Gynecol Clin North Am. 2009; 36(2):245-265.

15. Dieli-Conwright CM, Spektor TM, Rice JC, Schroeder ET. Hormone therapy attenuates exercise-induced skeletal muscle damage in postmenopausal women. J Appl Physiol. 2009;107(3):853858.

16. Chasse M, Tinmouth A, English SW, et al. Association of blood donor age and sex with recipient survival after red blood cell transfusion. JAMA Intern Med. 2016;176(9):1307-1314

17. Burger P, Hilarius-Stokman P, De Korte D, Van Den Berg TK, Van Bruggen R. CD47 functions as a molecular switch for erythrocyte phagocytosis. Blood. 2012;119(23):5512-5521.

18. Stewart A, Urbaniak S, Turner M, Bessos $H$. The application of a new quantitative assay for the monitoring of integrin-associated protein CD47 on red blood cells during storage and comparison with the expression of CD47 and phosphatidylserine with flow cytometry. Transfusion. 2005;45(9):1496-1503.

19. Patel KV, Mohanty JG, Kanapuru B, Hesdorffer C, Ershler WB, Rifkind JM. Association of the red cell distribution width with red blood cell deformability. Adv Exp Med Biol. 2013;765:211-216.

20. Ghezelbash B, Azarkeivan A, Pourfathollah AA, Deyhim M, Hajati E, Goodarzi A. Comparative evaluation of biochemical and hematological parameters of pre-storage leukoreduction during RBC storage. Int J Hematol Stem Cell Res. 2018;12(1):35-42. 